\title{
CONTROL OF CANCELLATIONS THAT RESTRAIN THE GROWTH OF A BINOMIAL RECURSION
}

\author{
MAGNUS ASPENBERG AND RODRIGO PÉREZ
}

\begin{abstract}
We study a recursion that generates real sequences depending on a parameter $x$. Given a negative $x$ the growth of the sequence is very difficult to estimate due to canceling terms. We reduce the study of the recursion to a problem about a family of integral operators, and prove that for every parameter value except -1 , the growth of the sequence is factorial. In the combinatorial part of the proof we show that when $x=-1$ the resulting recurrence yields the sequence of alternating Catalan numbers, and thus has exponential growth. We expect our methods to be useful in a variety of similar situations.
\end{abstract}

\section{INTRODUCTION}

Fix an arbitrary real number $x \neq 0$, and consider the sequence defined by the recursive expression

$$
a_{1}=x \quad, \quad a_{n}=x \sum_{r=\left\lceil\frac{n}{2}\right\rceil}^{n-1}\left(\begin{array}{c}
r \\
n-r
\end{array}\right) a_{r} .
$$

For $x=1$, formula (1) produces the sequence $1,2,7,34,214,1652, \ldots$ Note that the last summand $\left(\begin{array}{c}n-1 \\ 1\end{array}\right) a_{n-1}$ guarantees that $a_{n}>(n-1)$ ! This means that $\left\{a_{n}\right\}$ grows very fast since $n !>(n / e)^{n}$. We prove

Theorem 1. For any real $x \neq-1,0$ the sequence $\left\{a_{n}\right\}$ defined by (1) grows super-exponentially.

This is an interesting behavior, and not altogether obvious because when $x<0$, there are a lot of cancellations. In fact, when $x=-1$, the positive and negative terms exactly balance out to yield a surprising contrast:

Theorem 2. When $x=-1$, formula (1) produces the sequence $(-1)^{n} C_{n}$ of Catalan numbers with alternating signs, and therefore grows exponentially.

The phenomenon at play is very interesting. In the first part of the paper some combinatorial constructions will allow us to prove the results when $x>0$ and when $x \leq-1$. However, when $x \in(-1,0)$, the cancellations and the small size of $x$ conspire to render elementary arguments ineffective. In the second part we introduce functional analytic methods to control the effect of the cancellations in that case. We expect these ideas to be useful in a variety of similar situations.

1.1. Structure. The paper has two parts. In the first (sections 2 to 4 ) we prove Theorem 2 and the case $x \notin[-1,0]$ of Theorem 1 . Section 2 presents some basic facts about hypercube graphs and the Catalan numbers; Section 3 defines the combinatorial structure we use; and Section 4 contains the proofs.

The second author was supported by NSF grant DMS-0701557. 
The second part (sections 5 to 7 ) tackles the case $x \in(-1,0)$ of Theorem 1 . Section 5 uses the combinatorial knowledge gained in the first part to derive an alternative expression for $a_{n}$ as the sum of a sequence of numbers $S_{n}(1), \ldots, S_{n}(n-1)$ constructed recursively. This sequence is translated into a function $s_{n} \in L^{2}[0,1]$, and the recursion is interpreted as an integral operator. Section 6 contains the proof of the theorem assuming the statement of Lemma 10, and Section 7 is devoted to the proof of Lemma 10.

\section{Basic Combinatorial Facts}

2.1. Hypercubes. The hypercube graph $\mathcal{H}_{n}$ is the graph whose set of vertices $V_{n}$ consists of all $n$-vectors with coordinates 0 or 1 . Two vertices are adjacent whenever they differ in one coordinate. There is a natural stratification of $V_{n}$ by the number of coordinates of each value in a vertex; accordingly, let $V_{n, r} \subset V_{n}$ denote the vertices with $r$ coordinates equal to 1 . There are other equivalent definitions of hypercube graphs. The advantage of the definition in terms of binary coordinates is that the following facts become obvious; compare Figure 1.

(H1) $\left|V_{n}\right|=2^{n}$.

(H2) $\left|V_{n, r}\right|=\left(\begin{array}{l}n \\ r\end{array}\right)$.

(H3) If $n=m_{1}+m_{2}$, then $\mathcal{H}_{n}=\mathcal{H}_{m_{1}} \times \mathcal{H}_{m_{2}}$.

Incidentally, items (H1) and (H2) give a succinct proof of the binomial identity $\sum_{r=0}^{n}\left(\begin{array}{l}n \\ r\end{array}\right)=$ $2^{n}$. If instead of just counting vertices, they are assigned weight $x^{j}$, item (H3) furnishes a recursive proof of Newton's binomial formula

$$
\sum_{j=0}^{n}\left(\begin{array}{l}
n \\
r
\end{array}\right) x^{j}=(1+x)^{n}
$$
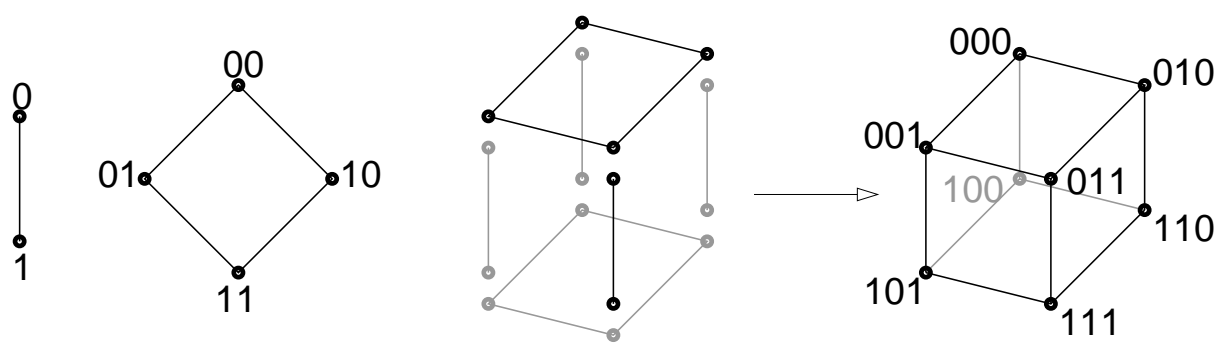

Figure 1. The hypercube graphs $\mathcal{H}_{1}, \mathcal{H}_{2}, \mathcal{H}_{3}$, and a decomposition of the latter as $\mathcal{H}_{1} \times \mathcal{H}_{2}$.

2.2. Catalan Numbers and Lattice Paths. The Catalan numbers $1,1,2,5,14,42,132, \ldots$ [5, A000108] are defined by the formula

$$
C_{n+1}=\frac{\left(\begin{array}{c}
2 n \\
n
\end{array}\right)}{(n+1)}
$$

The exponential rate of growth of the sequence $\left\{C_{n}\right\}$ follows easily from Stirling's formula:

$$
C_{n+1}=\frac{(2 n) !}{(n !)^{2}(n+1)} \sim \frac{\sqrt{2 \pi(2 n)}\left(\frac{2 n}{e}\right)^{2 n}}{\left(\sqrt{2 \pi n}\left(\frac{n}{e}\right)^{n}\right)^{2}(n+1)}=\frac{2^{2 n}}{\sqrt{\pi n}(n+1)} \sim \frac{4^{n}}{\sqrt{\pi} n^{3 / 2}} .
$$


Definition. A lattice path is a path in the lattice $\mathbb{Z} \times \mathbb{Z}$ that moves one horizontal or vertical unit at every step without self-intersections. We consider monotone paths, which never move left nor down. Note that a monotone path from $(0,0)$ to $(m, n)$ requires $m+n$ steps. Choosing one such path is tantamount to deciding which of these steps will be the $m$ horizontal steps, so the number of monotone lattice paths from $(0,0)$ to $(m, n)$ is $\left(\begin{array}{c}m+n \\ m\end{array}\right)$.

Lemma 3. The number of monotone paths from $(0,0)$ to $(n, n)$ that do not cross over the diagonal $\{y=x\}$ is equal to $C_{n}$.

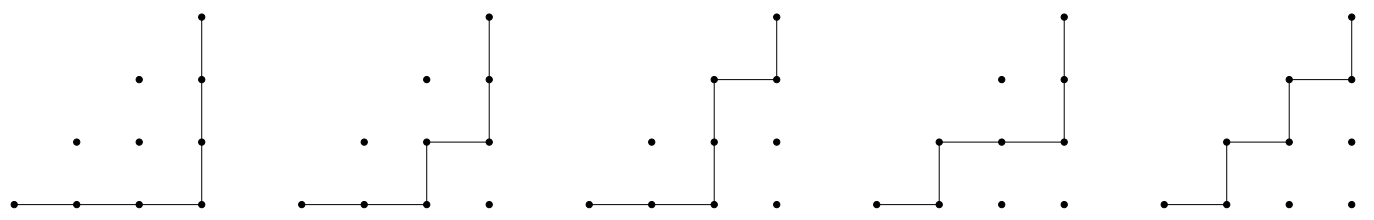

Figure 2. The $C_{3}=5$ monotone paths from $(0,0)$ to $(3,3)$.

Proof of Lemma 3. A monotone path $\gamma$ from $(0,0)$ to $(n, n)$ that crosses over the diagonal will pass through a point $(j, j+1)$. Let $P$ be the first such point, and $\gamma^{\prime}$ the portion of $\gamma$ going from $P$ to $(n, n)$. Reflecting $\gamma^{\prime}$ on the diagonal $\{y=x+1\}$ transforms $\gamma$ into a monotone path from $(0,0)$ to $(n-1, n+1)$. This operation is bijective because such paths must cross over the diagonal. Therefore the number of monotone paths from $(0,0)$ to $(n, n)$ that do not cross over the diagonal equals the number of all monotone paths from $(0,0)$ to $(n, n)$, minus the number of monotone paths from $(0,0)$ to $(n-1, n+1)$; i.e.,

$$
\left(\begin{array}{c}
2 n \\
n
\end{array}\right)-\left(\begin{array}{c}
2 n \\
n-1
\end{array}\right)=\left(\begin{array}{c}
2 n \\
n
\end{array}\right)-\frac{n}{n+1}\left(\begin{array}{c}
2 n \\
n
\end{array}\right)=\frac{\left(\begin{array}{c}
2 n \\
n
\end{array}\right)}{n+1} .
$$

3. StRUCTURE OF $a_{n}$

3.1. Signatures and Binomial Products. The internal structure of the expression $a_{n}$ is better understood by separating the different contributions of weight $x^{r}$. After expanding the recursive expressions in (1), the first few terms are

$$
\begin{aligned}
& a_{1}=\quad=x, \\
& a_{2}=x\left[\left(\begin{array}{l}
1 \\
1
\end{array}\right) a_{1}\right] \quad=\left(\begin{array}{l}
1 \\
1
\end{array}\right) x^{2}, \\
& a_{3}=x\left[\left(\begin{array}{l}
2 \\
1
\end{array}\right) a_{2}\right] \quad=\left(\begin{array}{l}
2 \\
1
\end{array}\right)\left(\begin{array}{l}
1 \\
1
\end{array}\right) x^{3}, \\
& a_{4}=x\left[\left(\begin{array}{l}
2 \\
2
\end{array}\right) a_{2}+\left(\begin{array}{l}
3 \\
1
\end{array}\right) a_{3}\right] \quad=\left(\begin{array}{l}
2 \\
2
\end{array}\right)\left(\begin{array}{l}
1 \\
1
\end{array}\right) x^{3}+\left(\begin{array}{l}
3 \\
1
\end{array}\right)\left(\begin{array}{l}
2 \\
1
\end{array}\right)\left(\begin{array}{l}
1 \\
1
\end{array}\right) x^{4}, \\
& a_{5}=x\left[\left(\begin{array}{l}
3 \\
2
\end{array}\right) a_{3}+\left(\begin{array}{l}
4 \\
1
\end{array}\right) a_{4}\right] \quad=\left(\begin{array}{l}
3 \\
2
\end{array}\right)\left(\begin{array}{l}
2 \\
1
\end{array}\right)\left(\begin{array}{l}
1 \\
1
\end{array}\right) x^{4}+\left(\begin{array}{l}
4 \\
1
\end{array}\right)\left(\begin{array}{l}
2 \\
2
\end{array}\right)\left(\begin{array}{l}
1 \\
1
\end{array}\right) x^{4}+ \\
& \left(\begin{array}{l}
4 \\
1
\end{array}\right)\left(\begin{array}{l}
3 \\
1
\end{array}\right)\left(\begin{array}{l}
2 \\
1
\end{array}\right)\left(\begin{array}{l}
1 \\
1
\end{array}\right) x^{5} \text {. }
\end{aligned}
$$

This symbolic manipulation makes it clear that $a_{n}$ is the sum of all products of the form

$$
\left(\begin{array}{c}
b_{s} \\
n-b_{s}
\end{array}\right)\left(\begin{array}{c}
b_{s-1} \\
b_{s-1}-b_{s-2}
\end{array}\right) \cdots\left(\begin{array}{c}
b_{2} \\
b_{2}-b_{1}
\end{array}\right)\left(\begin{array}{c}
b_{1} \\
1
\end{array}\right) \cdot x^{s+1},
$$

such that

$$
n=: b_{s+1}>b_{s}>b_{s-1}>\ldots>b_{1}=\underbrace{}_{3}, \quad \text { and } \quad b_{j+1} \leq 2 b_{j}(j=1, \ldots, s) .
$$


The last condition is a consequence of the fact that the sum in (1) starts at $r=\left\lceil\frac{n}{2}\right\rceil$. Note that this condition forces $b_{2}=2$.

Definition. A tuple $\sigma=\left(n, b_{s}, \ldots, b_{1}\right)$ satisfying (4) is called an $n$-signature. The $n$ signature that contains all the numbers from 1 to $n$ is called canonical.

Note. Compare the recursion (1) with the similar looking $\alpha_{1}=x, \alpha_{n}=x \sum_{r=\lceil n / 2\rceil}^{n-1} \alpha_{r}$ in which the binomial coefficients have been removed. From the above discussion we see that $\alpha_{n}$ is the sum of weights $x^{s}$, taken over all signatures $\left(n, b_{s}, \ldots, b_{1}\right)$. Replacing $x$ with 1 shows that the number of distinct $n$-signatures is given by the recursion

$$
N_{1}=1 \quad, \quad N_{n}=\sum_{r=\left\lceil\frac{n}{2}\right\rceil}^{n-1} N_{r} .
$$

The numbers $\left\{N_{n}\right\}=\{1,1,1,2,3,6,11,22,42, \ldots\}$ form the Narayana-Zidek-Capell sequence [5, A002083].

3.2. Arrays and blocks. When faced with an expression made of binomial coefficients, the natural thing to ask is what kind of combinatorial object is being counted. To a given signature $\sigma=\left(n, b_{s}, \ldots, b_{1}\right)$ we will assign a tower that can be filled with an array of numbers in exactly $\left(\begin{array}{c}b_{s} \\ n-b_{s}\end{array}\right) \ldots\left(\begin{array}{c}b_{1} \\ 1\end{array}\right)$ ways.

Definition. Given $\sigma=\left(n, b_{s}, \ldots, b_{1}\right)$, consider a tower of $n-1$ square cells split into blocks of lengths $\left(n-b_{s}\right),\left(b_{s}-b_{s-1}\right), \ldots,\left(b_{3}-b_{2}\right),\left(b_{2}-b_{1}\right)$ from top to bottom as in Figure 3 . The position of a block is the height $b_{j}$ of its lowest cell, so the signature condition $b_{j+1} \leq 2 b_{j}$ implies that a block is never taller than its position. An array associated to $\sigma$ is an assignment of numbers to every cell in the tower of $\sigma$ such that the numbers in the $j^{\text {th }}$ block (at position $b_{j}$ ) are chosen from the set $\left\{1,2, \ldots, b_{j}\right\}$ and appear in descending order. An array associated to the canonical signature is also called canonical.

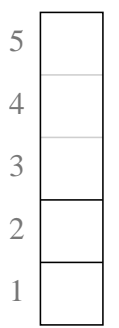

$(6,3,2,1)$

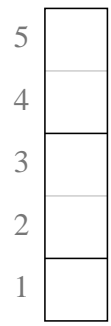

$(6,4,2,1)$

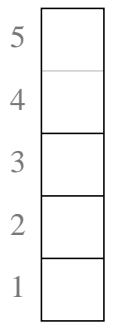

$(6,4,3,2,1)$

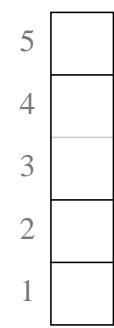

$(6,5,3,2,1)$

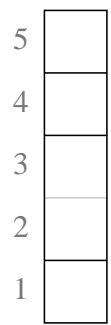

$(6,5,4,2,1)$

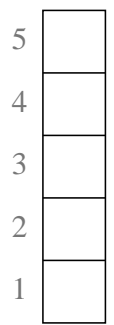

$(6,5,4,3,2,1)$

Figure 3. The towers associated to all 6-signatures. The second tower for instance, has blocks at positions 1,2 , and 4 . The rightmost signature is the canonical one.

Lemma 4. Let $\sigma=\left(n, b_{s}, \ldots, b_{1}\right)$ be an $n$-signature. Then

(a) The number of n-arrays associated to $\sigma$ is $\left(\begin{array}{c}b_{s} \\ n-b_{s}\end{array}\right) \ldots\left(\begin{array}{c}b_{1} \\ 1\end{array}\right)$.

(b) The number of canonical n-arrays is $(n-1)$ !

(c) The total number of n-arrays is given by formula (1) when $x=1$. 

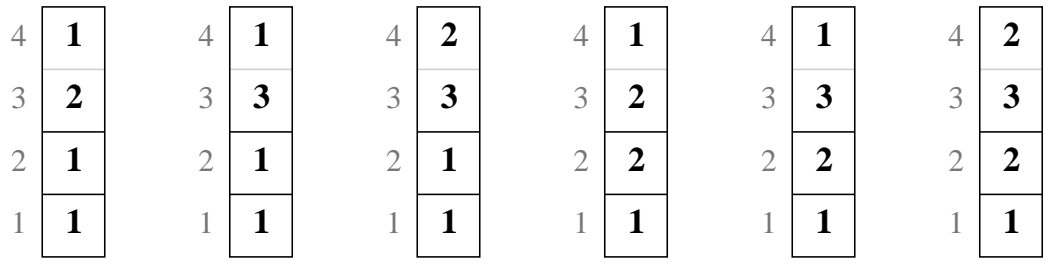

FiguRE 4 . The 5-signature $(5,3,2,1)$ has six associated arrays: The block at position 2 can hold either a 1 or a 2 , while the block at position 3 can hold any descending combination of the numbers $1,2,3$.

Proof. The tower associated to $\sigma$ has $s$ blocks. The $j^{\text {th }}$ block is based at position $b_{j}$ and its length is $b_{j+1}-b_{j}$ (for the topmost block the length is $n-b_{s}$ ). Therefore the $j^{\text {th }}$ block can be filled with an arbitrary choice of $b_{j+1}-b_{j}$ numbers between 1 and $b_{j}$; i.e., $\left(\begin{array}{c}b_{j} \\ b_{j+1}-b_{j}\end{array}\right)$ possibilities. This proves (a), from which item (b) follows immediately. To prove (c), note from (3) that $a_{n}$ counts $n$-arrays with weight $x^{s}$. Thus, when $x=1, a_{n}$ simply counts the number of $n$-arrays as claimed.

Definition. The sequence of numbers that specifies an array is called a pattern. We convene to read patterns from the bottom up; thus, for instance, the rightmost array in Figure 4 has pattern [1232].

Lemma 5. A tuple $\left[t_{1}, \ldots, t_{n-1}\right]$ is a valid pattern if and only if

$$
t_{j} \leq j \text { for all } 1 \leq j \leq n-1 .
$$

Proof. In a canonical array every block has length one. This means that the position of the $j^{\text {th }}$ block is $b_{j}=j$, and the number in this block is $t_{j}$. Thus, in this case, the pattern condition is equivalent to $t_{j} \leq b_{j}=j$, proving the result for canonical arrays.

In a non-canonical array, the cell at position $j$ belongs to a block at position $i \leq j$. The pattern condition states that the number $t_{j}$ in that cell must be at most $i$, and the result follows.

\subsection{Array Hypercubes.}

Definition. If an array has a block at position $p$ with more than one cell, the block can be split into two shorter blocks. The result is a valid array since the blocks have positions $p$ and $p+\eta>p$ ( $\eta$ is the location of the split within the original block), and the numbers in both blocks are all at most $p$. We call this operation on arrays a split. Note that an array can usually be split in several ways, all of which commute. Moreover, repeated splitting eventually results in a canonical array.

The reverse operation is also well defined. If an array has two consecutive blocks at positions $p$ and $p+\eta$, and the numbers contained in both blocks run together in descending order, the two blocks can be combined into a single one. This is because the new block is at position $p$ and contains numbers in descending order, which means that the length of the new block cannot exceed its position. In other words, condition (4) is satisfied. This operation on arrays is called a merge. As with splitting, merge operations are commutative, and repeated merging must terminate. An array where no pair of blocks can be merged is called primitive. 
Definition. The graph $\mathcal{G}_{n}$ on the set of $n$-arrays is defined by joining any two arrays related by a single split/merge operation. Arrays belong to the same connected component of $\mathcal{G}_{n}$ when they have the same pattern of numbers (disregarding block divisions). Note that a split/merge is possible at a given position if and only if the numbers at that position are in descending order. In particular, splitting/merging does not depend on the structure of blocks in an array, but only on the pattern of numbers. This yields the following lemma.

Lemma 6. Every connected component of $G_{n}$ is homeomorphic to a hypercube graph.

Proof. Consider an array $A \in \mathcal{G}_{n}$. The connected component $\mathcal{C}$ of $A$ consists of all arrays with the same pattern of numbers as $A$. This pattern has $\ell$ descents (locations where the numbers are in descending order). Now view such locations as placeholders for a symbol 1 or 0 depending on whether two blocks of $A$ meet at that location or not. This puts the arrays of $\mathcal{C}$ in correspondence with vertices of the hypercube graph $\mathcal{H}_{\ell}$; see Figure 5. Since a split/merge depends only on the pattern of numbers, all edges of $\mathcal{H}_{\ell}$ are included and $\mathcal{C}$ is homeomorphic to $\mathcal{H}_{\ell}$.

Observation. Every hypercube $\mathcal{C} \subset \mathcal{G}_{n}$ has a unique primitive array and a unique canonical array. In particular, the numbers of hypercubes in $\mathcal{G}_{n}$ and of primitive $n$-arrays are both equal to $(n-1)$ ! Also, if $\ell$ is as in the proof above, the primitive array has $s=n-\ell-1$ blocks (because the canonical array has $n-1$ ), so $\mathcal{C}$ is homeomorphic to $\mathcal{H}_{n-s-1}$.

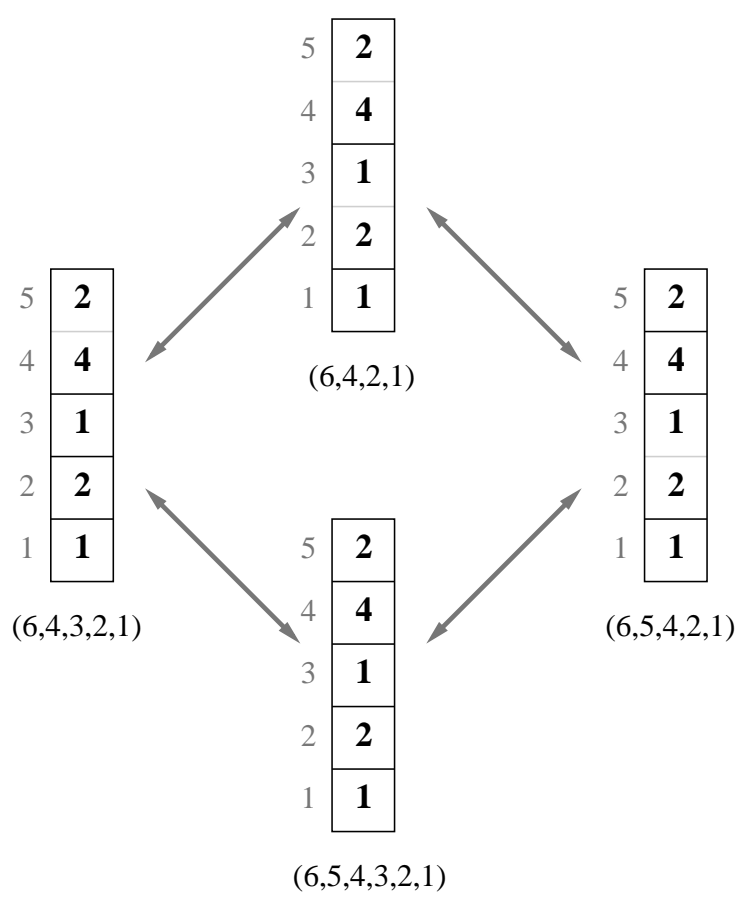

FiguRE 5. The pattern [12142] has 2 descents, and thus determines four 6 -arrays connected by split/merge operations into a square $\mathcal{H}_{2}$. 


\section{The First Proofs}

Each $a_{n}$ is a polynomial in $x$. When written with the monomials $\xi_{r} x^{r}$ in ascending order by degree, we say $a_{n}$ is in basic format. Since every $n$-array with $r-1$ blocks contributes $x^{r}$ to the total $a_{n}$, the coefficient $\xi_{r}$ counts the number of such arrays.

Example. The first few $a_{n}$ in basic format are (compare (2)):

$$
\begin{aligned}
& a_{1}=x, \\
& a_{2}=x^{2}, \\
& a_{3}=2 x^{3}, \\
& a_{4}=x^{3}+6 x^{4}, \\
& a_{5}=10 x^{4}+24 x^{5}, \\
& a_{6}=8 x^{4}+86 x^{5}+120 x^{6} .
\end{aligned}
$$

When $n=6$ for instance, we know that the towers with 4 blocks correspond to the three signatures $(6,4,3,2,1),(6,5,3,2,1)$, and $(6,5,4,2,1)$ (see Figure 3$)$. These have $\left(\begin{array}{l}4 \\ 2\end{array}\right)\left(\begin{array}{l}3 \\ 1\end{array}\right)\left(\begin{array}{l}2 \\ 1\end{array}\right)\left(\begin{array}{l}1 \\ 1\end{array}\right)=36,\left(\begin{array}{l}5 \\ 1\end{array}\right)\left(\begin{array}{l}3 \\ 2\end{array}\right)\left(\begin{array}{l}2 \\ 1\end{array}\right)\left(\begin{array}{l}1 \\ 1\end{array}\right)=30$, and $\left(\begin{array}{l}5 \\ 1\end{array}\right)\left(\begin{array}{l}4 \\ 1\end{array}\right)\left(\begin{array}{l}2 \\ 2\end{array}\right)\left(\begin{array}{l}1 \\ 1\end{array}\right)=20$ associated arrays respectively, and we see that each of these 86 arrays contributes $x^{5}$ to the value of $a_{6}$.

Recall that the array graph $\mathcal{G}_{n}$ consists of isolated hypercube components. We can also break $a_{n}$ down as a sum of contributions by hypercubes. Let $A$ be a primitive $n$-array with $s=r-1$ blocks. We know that $A$ contributes $x^{r}$ to $a_{n}$. Since the connected component $\mathcal{C}$ of $\mathcal{G}$ containing $A$ is homeomorphic to $\mathcal{H}_{n-r}$, items (H2) and (H4) in Section 2.1 give

$$
\sum_{a \in \mathcal{C}} x^{(\# \text { blocks of } a)+1} \stackrel{(\mathrm{H} 2)}{=} \sum_{j=0}^{n-r}\left(\begin{array}{c}
n-r \\
j
\end{array}\right) x^{r+j} \stackrel{(\mathrm{H} 4)}{=} x^{r}(1+x)^{n-r} .
$$

Let $\operatorname{prim}_{n}(r)$ be the number of primitive arrays with $r-1$ blocks; this is also the number of components of $\mathcal{G}_{n}$ homeomorphic to $\mathcal{H}_{n-r}$. Equation (6) shows that the total contribution to $a_{n}$ of all arrays in all such hypercubes is $\operatorname{prim}_{n}(r) \cdot x^{r}(1+x)^{n-r}$, so

$$
a_{n}=\sum_{r=\left\lceil\log _{2} n\right\rceil}^{n} \operatorname{prim}_{n}(r) \cdot x^{r}(1+x)^{n-r}
$$

(Condition (4) implies that the least possible number of blocks is $\left\lceil\log _{2} n\right\rceil$ ).

When $a_{n}$ is written in that form, we can read that each of the $\operatorname{prim}_{n}(r)$ components of $\mathcal{G}_{n}$ with dimension $n-r$ contributes $x^{r}(1+x)^{n-r}$ to the total sum $a_{n}$. We will say that $a_{n}$ is in binomial format.

Example. Let us compute the binomial format of $a_{6}$. From (5) we see that there are 8 arrays with 3 blocks. All of these must be primitive, so $\operatorname{prim}_{6}(4)=8$. These arrays (together with those obtained by splitting) determine 8 copies of $\mathcal{H}_{2}$ in $\mathcal{G}_{6}$. Gathering the monomials of all arrays in these hypercubes gives

$$
\begin{aligned}
a_{6}=8 x^{4}+86 x^{5}+120 x^{6}= & \\
& \left(8 x^{4}+16 x^{5}+8 x^{6}\right)+70 x^{5}+112 x^{6}= \\
& 8 x^{4}(1+x)^{2}+70 x^{5}+112 x^{6} .
\end{aligned}
$$


The remaining 70 arrays with 4 blocks must be primitive since they do not belong to an $\mathcal{H}_{2}$; i.e., $\operatorname{prim}_{6}(5)=70$. These arrays (together with those obtained by splitting) determine 70 copies of $\mathcal{H}_{1}$ in $\mathcal{G}_{6}$. Thus,

$$
8 x^{4}(1+x)^{2}+70 x^{5}+112 x^{6}=8 x^{4}(1+x)^{2}+70 x^{5}(1+x)^{1}+42 x^{6} .
$$

The first few $a_{n}$ in binomial format are:

$$
\begin{aligned}
& a_{1}=x^{1} y^{0}, \\
& a_{2}=x^{2} y^{0}, \\
& a_{3}=2 x^{3} y^{0}, \\
& a_{4}=x^{3} y^{1}+5 x^{4} y^{0}, \\
& a_{5}=10 x^{4} y^{1}+14 x^{5} y^{0}, \\
& a_{6}=8 x^{4} y^{2}+70 x^{5} y^{1}+42 x^{6} y^{0},
\end{aligned}
$$

where $y$ stand for $1+x$. This convetion makes for cleaner looking expressions, and will be consistently used in the rest of the paper.

Now that the combinatorial structure is in place, the proofs of Theorem 2 and the case $x \notin[-1,0]$ of Theorem 1 are straightforward.

Proof of Theorem 2. Since $x=-1$, the only non-zero term in (7) occurs when $r=n$. In other words,

$$
a_{n}=(-1)^{n} \operatorname{prim}_{n}(n) .
$$

Now, $\operatorname{prim}_{n}(n)$ is the number of $n$-arrays with $n-1$ blocks; that is, arrays that are both primitive and canonical. By definition, these are arrays with non-decreasing patterns of $n-1$ numbers. Any such pattern can be associated to a monotone lattice path from $(0,0)$ to $(n-1, n-1)$ that does not cross over the diagonal. Simply substract 1 from each entry in the pattern and interpret the results as heights of the horizontal steps of a monotone path (compare Figure 2). This procedure is bijective, so by Lemma 3, $\operatorname{prim}_{n}(n)=C_{n}$.

Proof of Theorem 1 when $x \notin[-1,0]$. Depending on the sign of $x$, one of the two formats for $a_{n}$ displays no cancellations.

$\underline{x>0}$ : All monomials $\xi_{r} x^{r}$ in the basic format of $a_{n}$ are positive because the coefficient $\xi_{r}$ counts arrays with $r-1$ blocks. In particular, $a_{n}$ is larger than the highest order monomial. The coefficient of this monomial is the number of arrays with the most blocks; i.e., canonical arrays. By Lemma 4,

$$
a_{n}>(n-1) ! \cdot x^{n}
$$

$\underline{x<-1}$ : Since $y<0$, all terms $\operatorname{prim}_{n}(r) \cdot x^{r} y^{n-r}$ in the binomial format of $a_{n}$ have the same sign, and do not cancel each other. Also, $|x|>|y|$, so

$$
\left|a_{n}\right|=\sum_{r} \operatorname{prim}_{n}(r) \cdot\left|x^{r} y^{n-r}\right|>\sum_{r} \operatorname{prim}_{n}(r) \cdot\left|y^{n}\right|=(n-1) ! \cdot\left|y^{n}\right|,
$$

where the last equality follows from the observation after Lemma 6 .

In both cases, $\left|a_{n}\right|$ is larger than $(n-1) ! \cdot w^{n}$ for some positive $w$, and the result holds. 


\section{THE CASE $x \in(-1,0)$}

The situation when $x \in(-1,0)$ is more delicate because the terms in both the basic and binomial formats of $a_{n}$ have alternating signs. Our strategy in this second part involves a different representation $((8)$ and $(9))$ of $a_{n}$. We will interpret the sequences $\left\{S_{n}(1), \ldots S_{n}(n-1)\right\}$ as functions $s_{n} \in L^{2}[0,1]$ and the recursion (9) as a sequence of integral operators $A_{n}: s_{n} \mapsto s_{n+1}$. We will deduce some facts about the shape of the graph of $s_{n}$, and about the $\operatorname{limit}$ operator $T=\lim A_{n}$. Then we will use this information to show that the largest eigenvalue $\lambda$ of $T$ bounds from below the exponential rate of decay of $a_{n} /(n-2)$ !

5.1. A new recursion. So far we have established that $a_{n}$ is the sum of contributions of the form $x^{r}$ over a large set of arrays (for each array, $r-1$ is the number of blocks). We grouped arrays with the same number pattern into hypercube graphs, and showed that $a_{n}$ is the sum of contributions $x^{n-\ell}(1+x)^{\ell}=x^{n-\ell} y^{\ell}$ over hypercubes $\mathcal{C}$, where $\ell$ is the dimension of each $\mathcal{C}$. This dimension is the number of descents in the associated pattern, so we can abandon arrays and express $a_{n}$ directly as a sum of contributions over patterns:

$$
a_{n}=\sum_{\ell=0}^{n-\left\lceil\log _{2} n\right\rceil-1} \sum_{\substack{\text { patterns with } \\ \ell \text { descents }}} x^{n-\ell} y^{\ell} .
$$

This allows us to sort the contributions to $a_{n}$ made by individual patterns. To this end, consider an $n$-pattern $\pi=\left[t_{1}, \ldots, t_{n-1}\right]$. If the truncated pattern $\left[t_{1}, \ldots, t_{n-2}\right]$ contributes $x^{a} y^{b}$ to $a_{n-1}$, then $\pi$ contributes $x^{a} y^{b+1}$ or $x^{a+1} y^{b}$ to $a_{n}$ depending on whether $t_{n-1}<t_{n-2}$ or not (i.e., on whether $\pi$ has one extra descent or not at the last position). This motivates the following definition.

Definition. For $n \geq 2$ let $S_{n}(r)$ denote the sum of contributions of all patterns $\left[t_{1}, \ldots, t_{n-1}\right]$ such that $t_{n-1}$ equals $r$ (thus, $r$ can take values in $\{1, \ldots, n-1\}$ ). In particular, $S_{2}(1)=x^{2}$, and

$$
a_{n}=\sum_{j=1}^{n-1} S_{n}(j) .
$$

By the previous argument, $S_{n+1}(r)$ can be computed from the contributions of $n$-patterns:

$$
S_{n+1}(r)=x \cdot \sum_{j=1}^{r} S_{n}(j)+y \cdot \sum_{j=r+1}^{n-1} S_{n}(j) .
$$

(when $r=n-1$ or $n$, there is no descent in the last position, so (9) should be interpreted to mean $\left.S_{n+1}(n-1)=S_{n+1}(n)=x \cdot \sum_{j=1}^{n-1} S_{n}(j)\right)$.

Figure 6 shows a plot of the values $\left\{S_{16}(1), \ldots, S_{16}(15)\right\}$ when $x=-1 / 2$. It is not coincidental that the graph looks sinusoidal.

5.2. Sinusoidal shape of $\boldsymbol{S}_{\boldsymbol{n}}$. Note that

$$
S_{n+1}(r)=S_{n+1}(r-1)-S_{n}(r) .
$$

From this relation we can derive a more convenient method of computing the sequence $S_{n}$ :

- $S_{2}(1)=x^{2} ;$ and for $n \geq 3$, 


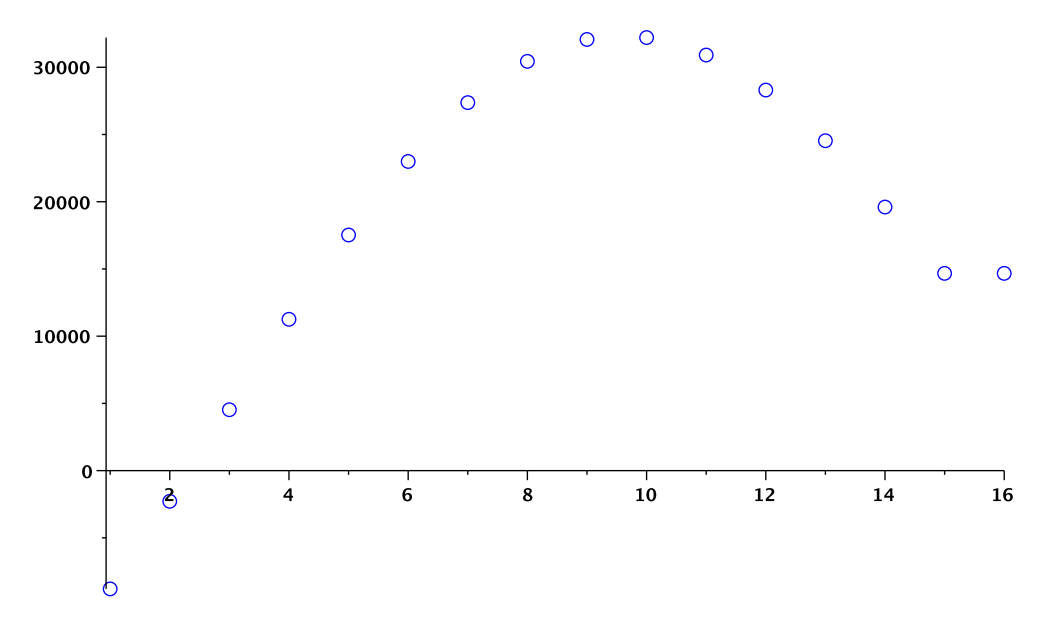

FigURE 6

- $S_{n}(0)=y \sum_{j=1}^{n-2} S_{n-1}(j)$,

- $S_{n}(r)=S_{n}(r-1)-S_{n-1}(r),(1 \leq r \leq n-2)$,

- $S_{n}(n-1)=S_{n}(n-2)$.

Observation. $S_{n}(0)$ is not part of the original sequence, but we will find it easier to study the properties of $S_{n}$ by including this auxiliary term in the discussion. For instance, note that $S_{n}(0)=y a_{n-1}$ and $S_{n}(n-1)=x a_{n-1}$. Recall that $y=1+x$ and $x \in(-1,0)$. It is vital to the coming arguments that these two values have opposite signs.

Definition. The sequence $S_{n}$ has

- a sign change if $n \geq 4$ and there are $a, b(1 \leq a<b \leq n-2)$ such that

$$
\begin{aligned}
& S_{n}(a)<0=S_{n}(a+1)=\ldots=S_{n}(b-1)<S_{n}(b) \text { (an up-change) or } \\
& S_{n}(a)>0=S_{n}(a+1)=\ldots=S_{n}(b-1)>S_{n}(b) \text { (a down-change), }
\end{aligned}
$$

- an extreme if $n \geq 5$ and there are $a, b(0<a<b-1 \leq n-3)$ such that

$$
\begin{aligned}
& S_{n}(a)<S_{n}(a+1)=\ldots=S_{n}(b-1)>S_{n}(b) \text { (a maximum) or } \\
& S_{n}(a)>S_{n}(a+1)=\ldots=S_{n}(b-1)<S_{n}(b) \text { (a minimum), }
\end{aligned}
$$

- an inflection if $n \geq 6$ and there are $a, b(1 \leq a<b-2 \leq n-4)$ such that

$$
\begin{aligned}
& 0<S_{n-1}(a+1)<S_{n-1}(a+2)=\ldots=S_{n-1}(b-1)>S_{n-1}(b)>0 \text { or } \\
& 0>S_{n-1}(a+1)>S_{n-1}(a+2)=\ldots=S_{n-1}(b-1)<S_{n-1}(b)<0 .
\end{aligned}
$$

The pair $(a, b)$ is the locus of the change/extreme/inflection. We also say that the change/extreme/inflection is located at $a$.

Observation. Recall that $S_{n-1}(c)=S_{n}(c+1)-S_{n}(c)$, so this value acts as a "discrete derivative" of the sequence $S_{n}$ in the definition of inflection. Notice that in our inflections the slope at the center is steeper than at the sides. A corollary of property (ShB) below is that no other inflection shape is necessary. Also, note that $S_{n}(n-1)$ is not allowed to be part of a change/extreme/inflection.

Proposition 7. For all $n \geq 6$ the sequence $S_{n}$ satisfies

(ShA) There are exactly one sign change, one extreme, and one inflection. 
(ShB) A maximum must have positive value and a minimum must have negative value.

(ShC) There is at most one $r$ such that $S_{n}(r)=0$.

(ShD) At least one of the two values $\min _{r} S_{n}(r)$ and $\max _{r} S_{n}(r)$ lies between $x a_{n}$ and $y a_{n}$.

(ShE) If the extreme has locus $(a, b)$ and the inflection has locus $(c, d)$, then

(a) $a \leq c \Longrightarrow S_{n-1}(0)<S_{n-1}(1)<S_{n-1}(2)<0$,

(b) $a>c \Longrightarrow 0>S_{n-1}(0)>S_{n-1}(1)>S_{n-1}(2)$.
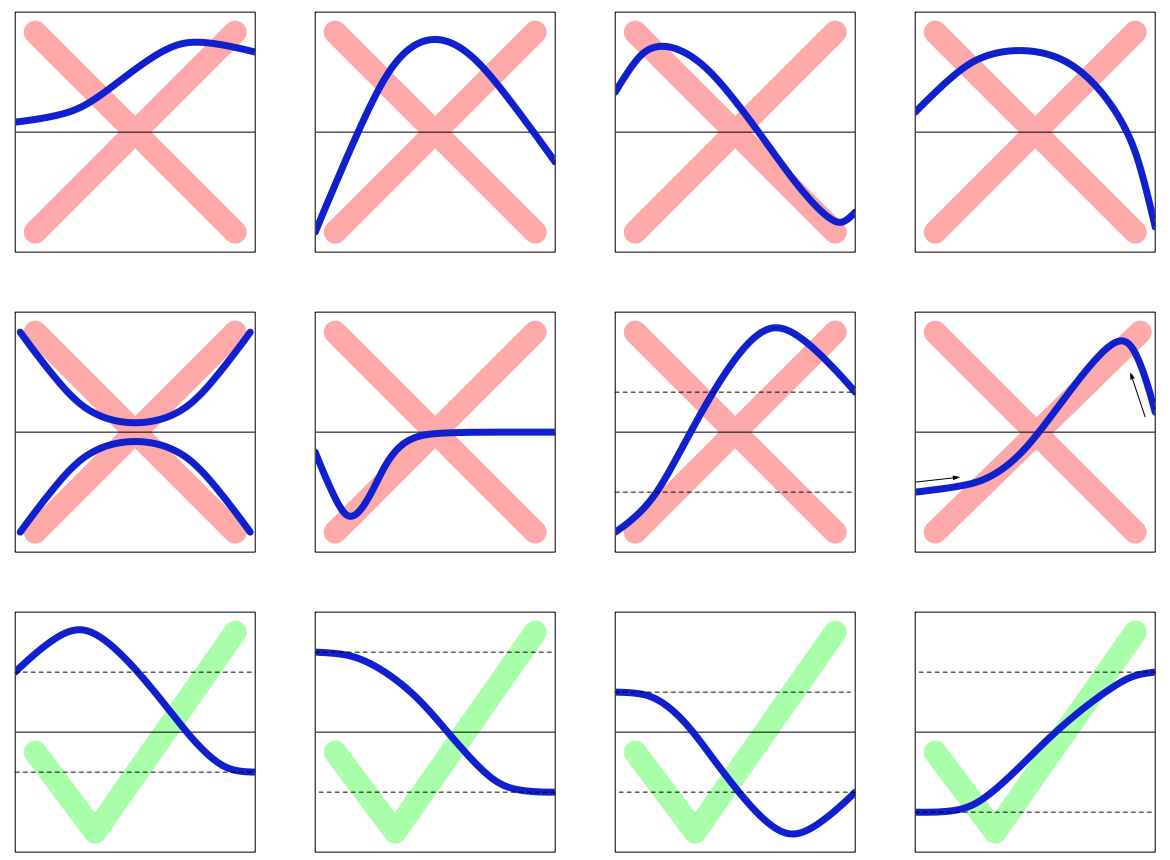

Figure 7. The first row of pictures illustrates property (ShA) of Proposition 7. (1): at least one zero. (2): at most one zero. (3): at most one extreme. (4): at least one inflection. The second row illustrates properties (ShB), (ShC), $(\mathrm{ShD})$, and $(\mathrm{ShE})$.

Proof of (ShA). A sign change in $S_{n}$ implies an extreme in $S_{n+1}$, which in turn implies an inflection in $S_{n+2}$; therefore we only need to prove that $S_{n}$ has exactly one sign change.

For $n \geq 4, S_{n}(0)=y a_{n-1}$ and $S_{n}(n-2)=x a_{n-1}$. Since $x$ and $y$ have opposite signs, the sequence $S_{n}$ has at least one sign change. If there were more changes than one, there would be at least three because $\operatorname{sgn}\left(S_{n}(0)\right) \neq \operatorname{sgn}\left(S_{n}(n-2)\right)$. Then $S_{n}$ has two extremes, and thus $S_{n-1}$ has two sign changes. But $S_{4}(2)=S_{4}(3)$, so $S_{4}$ can have at most one sign change, so by induction, $S_{n}$ has exactly one sign change.

Proof of $(S h B)$. Assume $S_{n}(n-1)>0$ (the negative case is analogous). In particular, a maximum of $S_{n}$ must lie above $S_{n}(n-1)$ and thus be positive. Also, $S_{n}(0)=y a_{n-1}<0$ because $S_{n}(n-1)=x a_{n-1}$.

○ If $S_{n}(1)<0$, then a minimum must lie lower than $S_{n}(1)$ and thus be negative.

○ If $S_{n}(1)>0$, then there is an increase from $S_{n}(0)$, so $S_{n-1}(1)<0$. Thus $S_{n-1}$ cannot have a down-change, and therefore $S_{n}$ cannot have a minimum. 
Proof of $(S h C)$. Since $S_{n}$ has at most one sign change, we need only discard the possibility of two (or more) consecutive zeros. Accordingly, assume that $S_{n}(r)=\ldots=S_{n}(r+k)=0$ $(k \geq 1)$ with $S_{n}(r-1)<0$ and $S_{n}(r+k+1) \neq 0$ (the case $S_{n}(r-1)>0$ is analogous).

Now, $S_{n}(r+k+1)<0$ contradicts $(\mathrm{ShB})$, so we can assume $S_{n}(r+k+1)>0$. But then

$$
S_{n-1}(r)<0=S_{n-1}(r+1)=\ldots=S_{n-1}(r+k)>S_{n-1}(r+k+1),
$$

and this means that $S_{n-1}$ contradicts $(\mathrm{ShB})$.

Proof of $(S h D)$. Assume $S_{n-1}(1)<0$ so $S_{n}(1)>y a_{n-1}$ (the case $S_{n-1}(1)>0$ is analogous). Moreover, $S_{n-1}$ cannot have a down-change (because it starts with a negative value); therefore $S_{n}$ cannot have a minimum. Since $S_{n}$ starts above $y a_{n-1}$ and ends at $x a_{n-1}$, the conclusion follows.

Proof of ( $S h E)$. We can assume that the extreme of $S_{n}$ is a maximum (the minimum case is analogous). Then $S_{n-1}$ has an up-change at $(a+1, b)$, and an extreme at $(c+1, d)$.

(a) If $a \leq c, S_{n-1}$ has an increase (namely the up-change) before its extreme. Hence the extreme is a maximum which, therefore, lies above $x a_{n-2}$. By (ShD),

$$
S_{n-1}(0)=y a_{n-2} \leq S_{n-1}(1) \leq S_{n-1}(2)<0 .
$$

(b) If $a>c, S_{n-1}$ has an increase (namely the up-change) after its extreme. Hence the extreme is a minimum which, therefore, lies below $y a_{n-2}$. In particular, $S_{n-1}$ is decreasing until this minimum. We claim that it is decreasing starting at the auxiliary term, i.e., that $S_{n-1}(0) \geq S_{n-1}(1)$; otherwise, $S_{n-2}$ has two sign changes. Then we have

$$
0>y a_{n}=S_{n-1}(0) \geq S_{n-1}(1) \geq S_{n-1}(2) .
$$

5.3. $\boldsymbol{S}_{\boldsymbol{n}}$ becomes a step function. Formula (9) induces a linear operator $\boldsymbol{A}_{n}: \mathbb{R}^{n-1} \longrightarrow$ $\mathbb{R}^{n}$. Here we will embed $\boldsymbol{A}_{n}$ as an integral operator $A_{n}: L^{2}[0,1] \longrightarrow L^{2}[0,1]$, and find an operator $T$ which is the limit of $\left\{A_{n}\right\}$ in the operator norm. The goal will be to link the growth of $\left\{a_{n}\right\}$ to the spectral properties of $T$.

For $n \geq 2$, the $r^{\text {th }}$ entry of the column vector

$$
\boldsymbol{s}_{n}=\left[\begin{array}{c}
S_{n}(1) /(n-2) ! \\
\vdots \\
S_{n}(n-1) /(n-2) !
\end{array}\right] \in \mathbb{R}^{n-1}
$$

represents the average contribution to $a_{n}$ of patterns with last entry $t_{n-1}=r$ (of which there are $(n-2)$ !). With this notation, Equation (9) can be interpreted as a linear transformation

$$
\boldsymbol{s}_{n+1}=\left(\boldsymbol{A}_{n} \cdot \boldsymbol{s}_{n}\right) /(n-1)
$$

where $\boldsymbol{A}_{n}$ is the $n \times(n-1)$ matrix whose $(i, j)$-entry is $x$ if $i \geq j$, and $y$ otherwise.

Let $E_{n}: \mathbb{R}^{n-1} \longrightarrow L^{2}[0,1]$ be the linear map that sends the standard basis vector $\boldsymbol{e}_{j}$ to the characteristic function of the interval $\left[\frac{j-1}{n-1}, \frac{j}{n-1}\right)$. The vector $\boldsymbol{s}_{n}$ maps to the step function $s_{n}=E_{n}\left(s_{n}\right)$, such that $s_{n}(u)=S_{n}(j) /(n-2)$ ! whenever $u \in\left[\frac{j-1}{n-1}, \frac{j}{n-1}\right)$. The maps $\left\{E_{n}\right\}$ 
embed the linear operators $\boldsymbol{A}_{n}: \mathbb{R}^{n-1} \longrightarrow \mathbb{R}^{n}$ into linear operators $A_{n}: L^{2}[0,1] \longrightarrow L^{2}[0,1]$. In particular, Equation (11) takes the form

$$
s_{n+1}(u)=\left[A_{n}\left(s_{n}\right)\right](u)=\int_{0}^{1} \alpha_{n}(u, v) \cdot s_{n}(v) \mathrm{d} v,
$$

where the kernel $\alpha_{n}$ is a piecewise constant function whose value at $(u, v) \in\left[\frac{i-1}{n}, \frac{i}{n}\right) \times$ $\left[\frac{j-1}{n-1}, \frac{j}{n-1}\right)$ is

$$
\alpha_{n}(u, v)=\left\{\begin{array}{ll}
x & \text { if } i \geq j \\
y & \text { otherwise }
\end{array} \quad \text { (i.e., equal to }\left(\boldsymbol{A}_{n}\right)_{i j}\right) .
$$

Observation. The factor $1 /(n-1)$ in (11) is hidden as a normalization factor in (12). Indeed, when $u$ lies in the interval $\left[\frac{i-1}{n}, \frac{i}{n}\right)$,

$$
\begin{aligned}
{\left[A_{n}\left(s_{n}\right)\right](u)=} & \int_{0}^{1} \alpha_{n}(u, v) \cdot E_{n}\left(\boldsymbol{s}_{n}\right)(v) \mathrm{d} v= \\
& \sum_{j=1}^{n-1} \int_{\left[\frac{j-1}{n-1}, \frac{j}{n-1}\right)}\left(\boldsymbol{A}_{n}\right)_{i j} \frac{S_{n}(j)}{(n-2) !} \mathrm{d} v= \\
& \sum_{j=1}^{n-1}\left(\boldsymbol{A}_{n}\right)_{i j} \frac{S_{n}(j)}{(n-2) !} \cdot \frac{1}{n-1},
\end{aligned}
$$

which is the $i^{\text {th }}$ entry of $\boldsymbol{s}_{n+1}$. In particular, (compare equation (8)):

$$
a_{n}=(n-1) ! \int_{0}^{1} s_{n}(v) \mathrm{d} v
$$

To prove the theorem we need to show that the rate of exponential decay of the integrals $\int_{0}^{1} s_{n}(v) \mathrm{d} v$ is bounded from below. The bound will be dictated by the largest eigenvalue $\lambda$ of the limit operator of $A_{n}$.

5.4. The limit operator $\boldsymbol{T}$. Here we define the limit operator $T$ of the sequence $\left\{A_{n}\right\}$, and establish some of its basic properties.

Let $T: L^{2}[0,1] \longrightarrow L^{2}[0,1]$ by

$$
(T f)(u)=\int_{0}^{1} \kappa(u, v) \cdot f(v) \mathrm{d} v
$$

with kernel

$$
\kappa(u, v)= \begin{cases}x & \text { if } u \geq v \\ y & \text { otherwise }\end{cases}
$$

Lemma 8. The operator $T$ is the limit of $\left\{A_{n}\right\}$ in the operator norm:

$$
\left\|T-A_{n}\right\| \leq \frac{1}{\sqrt{n}}
$$

Proof. The kernel of $T-A_{n}$ is the function $\kappa-\alpha_{n}$. Since $y$ is just a shorthand for $1+x$, we see that $\kappa-\alpha_{n}$ is the characteristic function of the staircase region $\Omega_{n}$ in the unit 


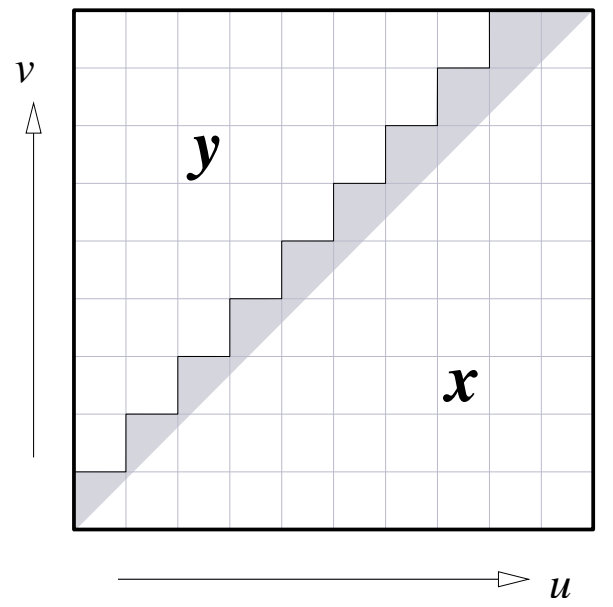

Figure 8 . The kernel $\alpha_{10}$ (the shaded region is $\Omega_{10}$ ). Note that the unit square is divided into rectangles of size $\frac{1}{10}$ by $\frac{1}{9}$.

square, consisting of the upper triangle $\{0 \leq u, v \leq 1 \mid u<v\}$, minus those rectangles $\left[\frac{i-1}{n}, \frac{i}{n}\right) \times\left[\frac{j-1}{n-1}, \frac{j}{n-1}\right)$ such that $i<j$. Then the Lebesgue measure of $\Omega_{n}$ is

$$
\mu\left(\Omega_{n}\right)=\frac{1}{2}-\frac{(n-2)(n-1)}{2} \cdot \frac{1}{n(n-1)}=\frac{1}{n} .
$$

It follows that

$$
\left\|T-A_{n}\right\|_{L^{2}} \leq\left(\mu\left(\Omega_{n}\right)\right)^{1 / 2}=\frac{1}{\sqrt{n}} .
$$

5.5. Eigenfunctions of $\boldsymbol{T}$. The operator $T$ can be expressed as follows:

$$
\begin{aligned}
(T f)(u)= & \int_{0}^{1} \kappa(u, v) \cdot f(v) \mathrm{d} v= \\
& x \cdot \int_{0}^{u} f(v) \mathrm{d} v+y \cdot \int_{u}^{1} f(v) \mathrm{d} v= \\
& -F(u)+y \cdot F(1)-x \cdot F(0),
\end{aligned}
$$

where $F$ is any primitive of $f$. To find the eigenvalues of $T$, set

$$
\begin{aligned}
(\lambda f)(u)= & (T f)(u)= \\
& -F(u)+y \cdot F(1)-x \cdot F(0),
\end{aligned}
$$

and differentiate to obtain the ODE

$$
f^{\prime}(u)=\frac{-f(u)}{\lambda},
$$

with general solution

$$
f(u)=C \mathrm{e}^{-u / \lambda} .
$$

A primitive of $f$ is $F(u)=-\lambda C \mathrm{e}^{-u / \lambda}$, so substituting in (13) gives

$$
\lambda\left(C \mathrm{e}^{-1 / \lambda}\right)=\lambda C \mathrm{e}^{-u / \lambda}-\lambda C\left(y \cdot \mathrm{e}^{-1 / \lambda}-x\right) .
$$


Thus, $\lambda$ is an eigenvalue if and only if

$$
\mathrm{e}^{-1 / \lambda}=\frac{x}{y}
$$

Note that $\frac{x}{y}<0$ exactly when $x \in(-1,0)$. Then we can write the eigenvalues as

$$
\lambda_{m}=\frac{-1}{\log \frac{x}{y}+2 m \pi \mathrm{i}}=\frac{-1}{\log \left|\frac{x}{y}\right|+(2 m+1) \pi \mathrm{i}} \text { for all } m \in \mathbb{Z},
$$

and the eigenfunction corresponding to $\lambda_{m}$ is

$$
f_{m}(u)=\left|\frac{x}{y}\right|^{u} \mathrm{e}^{(2 m+1) \pi \mathrm{i} u}
$$

Definition. For ease of notation, we write the absolute values of the two largest eigenvalues as $\lambda:=\left|\lambda_{-1}\right|=\left|\lambda_{0}\right|$ and $\mu:=\left|\lambda_{-2}\right|=\left|\lambda_{1}\right|$.

Lemma 9. The family of functions $\left\{f_{m}\right\}_{m \in \mathbb{Z}}$ forms a basis of $L^{2}[0,1]$.

Proof. Let $f$ be an arbitrary function in $L^{2}[0,1]$. Since $x \in(-1,0)$, the function $g(u)=$ $\left|\frac{y}{x}\right|^{u} \mathrm{e}^{-\pi \mathrm{i} u}$ is continuous, so $f g \in L^{2}[0,1]$. After rescaling the standard basis of $L^{2}[-\pi, \pi]$, we obtain the representation

$$
(f g)(u)=\sum_{m \in \mathbb{Z}} c_{m} \cdot \mathrm{e}^{2 m \pi \mathrm{i} u}
$$

which implies

$$
f(u)=\sum_{m \in \mathbb{Z}} c_{m} \cdot\left|\frac{x}{y}\right|^{u} \mathrm{e}^{(2 m+1) \pi \mathrm{i} u}=\sum_{m \in \mathbb{Z}} c_{m} \cdot f_{m}(u) .
$$

The reverse argument shows that $\left\{f_{m}\right\}$ are linearly independent.

In order to turn $\left\{f_{m}\right\}$ into an orthonormal basis, we introduce the weighted inner product

$$
\langle f, g\rangle=\int_{0}^{1}\left|\frac{x}{y}\right|^{-2 v} f(v) \bar{g}(v) \mathrm{d} v .
$$

Note that for $m \geq 0$ the pair of functions $f_{-(m+1)}, f_{m}$ are complex conjugate and their eigenvalues have the same magnitude. As a consequence, a convenient basis for the subspace $L_{\mathbb{R}}^{2}[0,1] \subset L^{2}[0,1]$ of real-valued functions is

$$
\left\{\left|\frac{x}{y}\right|^{u} \cos ((2 m+1) \pi u),\left|\frac{x}{y}\right|^{u} \sin ((2 m+1) \pi u)\right\}_{m \geq 0} .
$$

\section{The FunCtional APPROACH}

In this section we establish the lower bound on the exponential rate of decay of the sequence $a_{n} /(n-2)$ ! The long proof of Lemma 10 interferes with the flow of logic, and is consequently deferred to the next section.

Definition. The eigenfunctions $f_{-1}$ and $f_{0}$ with largest eigenvalue $\lambda$ span a complex twodimensional subspace of $L^{2}[0,1]$. Let $E \subset L_{\mathbb{R}}^{2}[0,1]$ denote the real slice of this subspace generated by $\left\{\left|\frac{x}{y}\right|^{u} \cos (\pi u),\left|\frac{x}{y}\right|^{u} \sin (\pi u)\right\}$. The space $E^{\perp}$ spanned by all other eigenfunctions is orthogonal to $E$, so that (by Lemma 9) $L_{\mathbb{R}}^{2}[0,1]=E \oplus E^{\perp}$. The projections onto $E$ 
and $E^{\perp}$ are denoted $P$ and $P^{\perp}$ respectively. By Parseval's Theorem we can define the angle $\theta_{n}$ by any of the three equivalent formulas

$$
\sin \theta_{n}:=\frac{\left\|P^{\perp} s_{n}\right\|_{2}}{\left\|s_{n}\right\|_{2}}, \quad \cos \theta_{n}:=\frac{\left\|P s_{n}\right\|_{2}}{\left\|s_{n}\right\|_{2}}, \quad \tan \theta_{n}:=\frac{\left\|P^{\perp} s_{n}\right\|_{2}}{\left\|P s_{n}\right\|_{2}} .
$$

Intuitively, the closer $\theta_{n}$ is to 0 , the better $s_{n}$ resembles a function in $E$.

Now we can describe the strategy of the proof:

Step 1: We use the shape properties of the sequence $S_{n}$ to show that the angles $\theta_{n}$ are bounded away from $\pi / 2$.

Step 2: The sequence $\left\{\theta_{n}\right\}$ converges to 0 , so the functions $s_{n}$ become progressively sinusoidal. Step 3: There is a sequence of indices $\left\{n_{k}\right\}$ such that $\left\{\left|a_{n_{k}}\right|\right\}$ is comparable to $\left\{\left\|s_{n_{k}}\right\|_{2}\right\}$. Meanwhile, $\left\|s_{n}\right\|_{2} \geq(\lambda-\varepsilon)^{n}$ for arbitrarily small $\varepsilon$, and the result will follow.

6.1. Step $1\left(\boldsymbol{\theta}_{\boldsymbol{n}} \leq \boldsymbol{\Theta}<\boldsymbol{\pi} / \mathbf{2}\right)$. Fix $n$ and consider the locus $(a, b)$ of the sign change of $S_{n}$. The value $z_{n}:=a /(n-1) \in[0,1)$ is such that $s_{n}(u) \cdot \sin \left(\pi\left(u-z_{n}\right)\right)$ never changes sign. We will show there is a $K>0$ such that for all $n$,

$$
\mid\left\langle s_{n}(u),\left|\frac{x}{y}\right|^{u} \sin \pi\left(u-z_{n}\right\rangle\right|>K\left\|s_{n}\right\|_{2} .
$$

The projection $P s_{n}$ is larger than $\mid\left\langle s_{n}(u),\left|\frac{x}{y}\right|^{u} \sin \pi\left(u-z_{n}\right\rangle\right|$, and thus the angle $\theta_{n}$ is bounded away from $\pi / 2$ by

$$
\theta_{n}<\arccos K=: \Theta<\pi / 2 \text {. }
$$

The proof of (15) follows from Corollary 11 and Lemma 12 below, using $K=C C^{\prime}$.

Lemma 10. The sequences $\left\{\left\|s_{n}\right\|_{1}\right\}$ and $\left\{\left\|s_{n}\right\|_{\infty}\right\}$ are comparable in the sense that there is a constant $C$ such that for all $n$,

$$
\left\|s_{n}\right\|_{\infty} \geq\left\|s_{n}\right\|_{1} \geq C\left\|s_{n}\right\|_{\infty} .
$$

This is the main technical lemma, and its proof is deferred to Section 7.

Corollary 11. The sequences $\left\{\left\|s_{n}\right\|_{1}\right\}$ and $\left\{\left\|s_{n}\right\|_{2}\right\}$ are comparable in the sense that there is a constant $C$ such that for all $n$,

$$
\left\|s_{n}\right\|_{2} \geq\left\|s_{n}\right\|_{1} \geq C\left\|s_{n}\right\|_{2} .
$$

Proof. The left side is the Cauchy-Schwarz inequality. On the right we have

$$
\left\|s_{n}\right\|_{1}^{2} \geq C^{2}\left\|s_{n}\right\|_{\infty}^{2} \geq C^{2} \int_{0}^{1} s_{n}^{2}(v) \mathrm{d} v=C^{2}\left\|s_{n}\right\|_{2}^{2}
$$

Lemma 12. Let $z_{n}$ be defined as above. Then there is a constant $C^{\prime}$ such that

$$
\left|\left\langle s_{n}(u),\left|\frac{x}{y}\right|^{u} \sin \pi\left(u-z_{n}\right)\right\rangle\right|>C^{\prime}\left\|s_{n}\right\|_{1} .
$$

Proof. Given $z \in[0,1]$, the maximum of the function $\left|\frac{x}{y}\right|^{-u} \cdot|\sin \pi(u-z)|$ in a small interval $[z-\varepsilon, z+\varepsilon] \cap[0,1]$ is $M_{z, \varepsilon}>0$; a quantity that varies continuously. Fix $\varepsilon \equiv \varepsilon(z)$ so that the Lebesgue measure of the set

$$
L_{z}:=\left\{u \in[0,1] \text { s.t. }\left|\frac{x}{y}\right|^{-u} \cdot|\sin \pi(u-z)|<M_{z, \varepsilon}\right\}
$$


is $C / 2$, where $C$ is the constant of Lemma 10. This is well defined because $\left|\frac{x}{y}\right|^{-u} \cdot|\sin \pi(u-z)|$ is nowhere constant, so the measure of $L_{z}$ varies continuously. Since $[0,1]$ is compact, the lower bound $M:=\inf _{z \in[0,1]} M_{z, \varepsilon}$ is positive.

Recall that $z_{n}$ is chosen so that $s_{n}(u) \cdot \sin \pi\left(u-z_{n}\right)$ has constant sign. For simplicity, let us assume that this sign is positive. Then the weighted inner product $\left|\left\langle s_{n}(u),\left|\frac{x}{y}\right|^{u} \sin \pi\left(u-z_{n}\right)\right\rangle\right|$ equals (compare (14)):

$$
\begin{gathered}
\int_{0}^{1}\left|\frac{x}{y}\right|^{-v} s_{n}(v) \cdot \sin \pi\left(v-z_{n}\right) \mathrm{d} v \geq \int_{L_{z_{n}}^{\complement}}\left|\frac{x}{y}\right|^{-v} s_{n}(v) \cdot \sin \pi\left(v-z_{n}\right) \mathrm{d} v> \\
M \int_{L_{z_{n}}^{\complement}}\left|s_{n}(v)\right| \mathrm{d} v=M\left(\int_{0}^{1}\left|s_{n}(v)\right| \mathrm{d} v-\int_{L_{z_{n}}}\left|s_{n}(v)\right| \mathrm{d} v\right) .
\end{gathered}
$$

But

$$
\int_{L_{z_{n}}}\left|s_{n}(v)\right| \mathrm{d} v<\mu\left(L_{z_{n}}\right)\left\|s_{n}\right\|_{\infty}=\frac{C}{2}\left\|s_{n}\right\|_{\infty}<\frac{1}{2}\left\|s_{n}\right\|_{1}
$$

by Lemma 10, so we get

$$
\left|\left\langle s_{n}(u),\left|\frac{x}{y}\right|^{u} \sin \pi\left(u-z_{n}\right)\right\rangle\right|>\frac{M}{2}\left\|s_{n}\right\|_{1} .
$$

6.2. Step $2\left(\boldsymbol{\theta}_{\boldsymbol{n}} \rightarrow \mathbf{0}\right)$. We will show in Lemma 13 that when $n$ is large enough, the sequence $\left\{\tan \theta_{n}\right\}$ enters a decreasing regime that makes it eventually converge to 0 . This establishes the desired result.

First we derive two versions of the basic estimate for $\tan \theta_{n+1}$ :

$$
\tan \theta_{n+1}=\frac{\left\|P^{\perp} A_{n} s_{n}\right\|_{2}}{\left\|P A_{n} s_{n}\right\|_{2}}=\frac{\left\|P^{\perp}\left[T s_{n}+\left(A_{n}-T\right)\right]\right\|_{2}}{\left\|P\left[T s_{n}+\left(A_{n}-T\right)\right]\right\|_{2}} \leq \frac{\left\|P^{\perp} T s_{n}\right\|_{2}+\left\|P^{\perp}\left(A_{n}-T\right) s_{n}\right\|_{2}}{\left|\left\|P T s_{n}\right\|_{2}-\left\|P\left(A_{n}-T\right) s_{n}\right\|_{2}\right|},
$$

and that $\theta_{n}<\Theta$ allows us to remove the absolute value in the denominator by assuming $n$ is large enough. Since $T$ commutes with the projections $P$ and $P^{\perp}$, and using Lemma 8,

$$
\begin{aligned}
\tan \theta_{n+1} & \leq \frac{\mu\left\|P^{\perp} s_{n}\right\|_{2}+\left\|s_{n}\right\|_{2} / \sqrt{n}}{\lambda\left\|P s_{n}\right\|_{2}-\left\|s_{n}\right\|_{2} / \sqrt{n}}= \\
& =\frac{\sqrt{n} \mu \sin \theta_{n}+1}{\sqrt{n} \lambda \cos \theta_{n}-1} \\
& =\left(\frac{\mu+\frac{1}{\sqrt{n} \sin \theta_{n}}}{\lambda-\frac{1}{\sqrt{n} \cos \theta_{n}}}\right) \tan \theta_{n} .
\end{aligned}
$$

Lemma 13. Let $n>\frac{9}{\cos ^{2} \Theta(\lambda-\mu)^{2}}$. Then there are constants $0<\varepsilon<1$ and $R>0$ such that a) If $\sqrt{n} \sin \theta_{n} \geq \frac{3}{\lambda-\mu}$, then

$$
\tan \theta_{n+1}<(1-\varepsilon) \tan \theta_{n}
$$

b) If $\sqrt{n} \sin \theta_{n} \leq \frac{3}{\lambda-\mu}$, then

$$
\tan \theta_{n+1}<\frac{R}{\sqrt{n}}
$$

In other words, when $n$ is sufficiently large, each step in the sequence $\left\{\tan \theta_{n}\right\}$ affords a definite relative decrease, or a slower but absolute decrease. 
Proof of Lemma 13.

a) The angle $\theta_{n}$ is smaller than $\Theta$, so the initial assumption on $n$ implies

$$
\lambda-\frac{1}{\sqrt{n} \cos \theta_{n}}>\lambda-\frac{1}{\sqrt{n} \cos \Theta}>\lambda-\frac{\lambda-\mu}{3} .
$$

The condition $\sqrt{n} \sin \theta_{n} \geq \frac{3}{\lambda-\mu}$ is equivalent to

$$
\mu+\frac{1}{\sqrt{n} \sin \theta_{n}}<\mu+\frac{\lambda-\mu}{3}
$$

so (17) is smaller than

$$
\left(\frac{\mu+\frac{\lambda-\mu}{3}}{\lambda-\frac{\lambda-\mu}{3}}\right) \tan \theta_{n}=\left(\frac{2 \mu+\lambda}{2 \lambda+\mu}\right) \tan \theta_{n} .
$$

Since $\frac{2 \mu+\lambda}{2 \lambda+\mu}<1$, this case is proved.

b) When $\sqrt{n} \sin \theta_{n} \leq \frac{3}{\lambda-\mu}$, we also have $\sqrt{n} \cos \theta_{n} \geq \sqrt{n-\frac{9}{(\lambda-\mu)^{2}}}$. Substituting in (16) gives

$$
\tan \theta_{n+1}<\frac{\mu \frac{3}{\lambda-\mu}+1}{\lambda \sqrt{n-\frac{9}{(\lambda-\mu)^{2}}}-1},
$$

and the result follows.

6.3. Step $3\left(\left|\int s_{n_{k}}\right|>\operatorname{cst}(\lambda-\varepsilon)^{n_{k}}\right)$. We are ready to prove that

$$
\left|\int_{0}^{1} s_{n}(v) \mathrm{d} v\right| \geq(\lambda-\varepsilon)^{n}
$$

along a subsequence of indices. The immediate consequence is factorial growth of $\left|a_{n}\right|$, since $a_{n}=(n-2) ! \cdot \int_{0}^{1} s_{n}(v) \mathrm{d} v$. Equation (18) follows at once from propositions 14 and 15.

Proposition 14. There is an infinite integer sequence $n_{1}<n_{2}<\ldots$, and a constant $0<W \leq 1$ such that for all $k$,

$$
\left|\int_{0}^{1} s_{n_{k}}(v) \mathrm{d} v\right| \geq \frac{W}{\sqrt{17}}\left\|s_{n_{k}}\right\|_{2} .
$$

Proposition 15. For every $\varepsilon>0$ there are constants $G, N>0$ such that for $n>N$,

$$
\left\|s_{n}\right\|_{2}>G(\lambda-\varepsilon)^{n} .
$$

The proof of Proposition 14 uses the following auxiliary result:

Lemma 16. There is an infinite integer sequence $n_{1}<n_{2}<\ldots$ such that for all $k$,

$$
\left|\int_{0}^{1} P s_{n_{k}}(v) \mathrm{d} v\right| \geq \frac{1}{2} \int_{0}^{1}\left|P s_{n_{k}}(v)\right| \mathrm{d} v .
$$

We prove Lemma 16 first, and then propositions 14 and 15. 
Proof of Lemma 16. First notice that $\int_{0}^{1} \sin (\pi(u+\omega)) \mathrm{d} v=\frac{2}{\pi} \cos \pi \omega$, so

$$
\int_{0}^{1} \sin (\pi(u+\omega)) \mathrm{d} v \geq \frac{1}{\sqrt{2}} \int_{0}^{1} \sin (\pi u) \mathrm{d} v \text { if and only if }|\omega(\bmod 1)| \leq 1 / 4 .
$$

The lemma will follow once we prove that if a large enough $n$ does not satisfy (19), then $n+1$ does. Accordingly, assume that

$$
\left|\int_{0}^{1} P s_{n}(v) \mathrm{d} v\right| \leq \frac{1}{2} \int_{0}^{1}\left|P s_{n}(v)\right| \mathrm{d} v<\frac{1}{\sqrt{2}} \int_{0}^{1}\left|P s_{n}(v)\right| \mathrm{d} v .
$$

Since the function $P s_{n}$ is in $E$, it has the form $P s_{n}(u)=B \sin (\pi(u+\omega))$ for some constants $B, \omega$. According to $(20)$, the assumption above means that $|\omega(\bmod 1)| \geq 1 / 4$. Now,

$$
\left|\int_{0}^{1} P s_{n+1}(v) \mathrm{d} v\right|=\left|\int_{0}^{1} P T s_{n}(v) \mathrm{d} v+\int_{0}^{1} P\left(A_{n}-T\right) s_{n}(v) \mathrm{d} v\right| .
$$

We estimate both terms on the right side. On one hand, since $P$ commutes with $T$, and $|(\omega+1 / 2)(\bmod 1)| \leq 1 / 4$

$$
\begin{gathered}
\left|\int_{0}^{1} P T s_{n}(v) \mathrm{d} v\right|=\left|\int_{0}^{1} \lambda B \sin (\pi(v+\omega+1 / 2)) \mathrm{d} v\right| \geq \\
\frac{\lambda}{\sqrt{2}} \int_{0}^{1}|B \sin (\pi(u+\omega+1 / 2))| \mathrm{d} v=\frac{\lambda}{\sqrt{2}} \int_{0}^{1}|B \sin (\pi(u+\omega))| \mathrm{d} v=\frac{\lambda}{\sqrt{2}}\left\|P s_{n}\right\|_{1} .
\end{gathered}
$$

On the other hand,

$$
\begin{gathered}
\left|\int_{0}^{1} P\left(A_{n}-T\right) s_{n}(v) \mathrm{d} v\right| \leq \int_{0}^{1}\left|P\left(A_{n}-T\right) s_{n}(v)\right| \mathrm{d} v= \\
\left\|P\left(A_{n}-T\right) s_{n}\right\|_{1} \leq\left\|P\left(A_{n}-T\right) s_{n}\right\|_{2} \leq\left\|\left(A_{n}-T\right) s_{n}\right\|_{2} \leq \frac{1}{\sqrt{n}}\left\|s_{n}\right\|_{2}
\end{gathered}
$$

by Lemma 8 . Since $\theta_{n}<\Theta<\pi / 2$, the last quantity is smaller than

$$
\frac{1}{\cos \Theta \sqrt{n}}\left\|P s_{n}\right\|_{2} \leq \frac{1}{\cos \Theta \sqrt{n}} \frac{2 \sqrt{2}}{\pi}\left\|P s_{n}\right\|_{1},
$$

where the last estimate comes from comparing the 1- and 2-norms of a sine function. Altogether, plugging both estimates in (21) gives

$$
\left|\int_{0}^{1} P s_{n+1}(v) \mathrm{d} v\right| \geq\left(\frac{\lambda}{\sqrt{2}}-\frac{2 \sqrt{2}}{\pi \cos \Theta \sqrt{n}}\right)\left\|P s_{n}\right\|_{1} .
$$

It only rests to compare $\left\|P s_{n}\right\|_{1}$ with $\left\|P s_{n+1}\right\|_{1}=\left\|P T s_{n}+P\left(A_{n}-T\right) s_{n}\right\|_{1}$. Since $P$ and $T$ commute, $\left\|P T s_{n}\right\|_{1}=\lambda\left\|P s_{n}\right\|_{1}$. Also, $\left\|P\left(A_{n}-T\right) s_{n}\right\|_{1} \leq \frac{2 \sqrt{2}}{\pi \cos \Theta \sqrt{n}}\left\|P s_{n}\right\|_{1}$, as we saw above. This gives

$$
\left\|P s_{n}\right\|_{1} \geq\left(\lambda+\frac{2 \sqrt{2}}{\pi \cos \Theta \sqrt{n}}\right)^{-1}\left\|P s_{n+1}\right\|_{1},
$$

which, plugged back in (22) gives

$$
\left|\int_{0}^{1} P s_{n+1}(v) \mathrm{d} v\right| \geq\left(\frac{\frac{\lambda}{\sqrt{2}}-\frac{2 \sqrt{2}}{\pi \cos \Theta \sqrt{n}}}{\lambda+\frac{2 \sqrt{2}}{\pi \cos \Theta \sqrt{n}}}\right)\left\|P s_{n+1}\right\|_{1} .
$$

For large enough $n$ the last quantity is larger than $\frac{1}{2}\left\|P s_{n+1}\right\|_{1}$, and the result follows.

With the above result we are ready to prove propositions 14 and 15, establishing (18) and our main result. 
Proof of Proposition 14. Consider the sequence $\left\{n_{k}\right\}$ from Lemma 16, truncated in the beginning so that the right hand expression in

$$
\begin{aligned}
\left|\int_{0}^{1} s_{n_{k}}(v) \mathrm{d} v\right|=\left|\int_{0}^{1} P s_{n_{k}}(v) \mathrm{d} v+\int_{0}^{1} P^{\perp} s_{n_{k}}(v) \mathrm{d} v\right| \geq & \\
& \left|\int_{0}^{1} P s_{n_{k}}(v) \mathrm{d} v\right|-\left|\int_{0}^{1} P^{\perp} s_{n_{k}}(v) \mathrm{d} v\right|
\end{aligned}
$$

is positive. Let us evaluate both terms. By Lemma 16,

$$
\left|\int_{0}^{1} P s_{n_{k}}(v) \mathrm{d} v\right| \geq \frac{1}{2}\left\|P s_{n_{k}}\right\|_{1} \geq \frac{W}{2}\left\|P s_{n_{k}}\right\|_{2}
$$

where $0<W \leq 1$ is a lower bound on the quotient of the 1- and 2-norms of $\left|\frac{x}{y}\right|^{u} \sin \pi(u+\phi)$ (here $0 \leq \phi \leq 1$ is an arbitrary phase shift). On the other hand, the triangle and CauchySchwarz inequalities give

$$
\left|\int_{0}^{1} P^{\perp} s_{n_{k}}(v) \mathrm{d} v\right| \leq\left\|P^{\perp} s_{n_{k}}\right\|_{2} .
$$

If $n_{k}$ is sufficiently large, then $\tan \theta_{n_{k}}<W / 4 \leq 1 / 4$, where $W$ is as before, and we get

$$
\left\|P^{\perp} s_{n_{k}}\right\|_{2}=\tan \theta_{n_{k}}\left\|P s_{n_{k}}\right\|_{2} \leq \frac{W}{4}\left\|P s_{n_{k}}\right\|_{2} .
$$

Plugging these estimates back in (23) gives

$$
\left|\int_{0}^{1} s_{n_{k}}(v) \mathrm{d} v\right| \geq \frac{W}{2}\left\|P s_{n_{k}}\right\|_{2}-\frac{W}{4}\left\|P s_{n_{k}}\right\|_{2}=\frac{W}{4}\left\|P s_{n_{k}}\right\|_{2}=\frac{W}{4} \cos \theta_{n_{k}}\left\|s_{n_{k}}\right\|_{2}>\frac{W}{\sqrt{17}}\left\|s_{n_{k}}\right\|_{2},
$$

since $\tan \theta_{n_{k}}<1 / 4$.

Proof of Proposition 15. For large enough $n$, the right hand side of

$$
\left\|s_{n+1}\right\|_{2}=\left\|T s_{n}+\left(A_{n}-T\right) s_{n}\right\|_{2} \geq\left\|T s_{n}\right\|_{2}-\left\|\left(A_{n}-T\right) s_{n}\right\|_{2}
$$

is positive. In fact, the two terms on the right have the bounds

$$
\left\|T s_{n}\right\|_{2} \geq\left\|T P s_{n}\right\|_{2}=\lambda \cos \theta_{n}\left\|s_{n}\right\|_{2},
$$

and

by Lemma 8, so

$$
\left\|\left(A_{n}-T\right) s_{n}\right\|_{2} \leq \frac{1}{\sqrt{n}}\left\|s_{n}\right\|_{2}
$$

$$
\left\|s_{n+1}\right\|_{2} \geq\left(\lambda \cos \theta_{n}-\frac{1}{\sqrt{n}}\right)\left\|s_{n}\right\|_{2} .
$$

Since $\theta_{n}<\Theta$, the result follows.

\section{Proof of Lemma 10}

The inequality $\left\|s_{n}\right\|_{1} \leq\left\|s_{n}\right\|_{\infty}$ is trivial, but the opposite direction requires estimates based on the shape of the sequences $S_{n}$. Because of the rescaling

$$
\frac{S_{n}(j)}{(n-2) !}=s_{n}\left(\frac{j-1 / 2}{n-1}\right),
$$

all statements about the shape of the sequence $S_{n}$ can be interpreted as applying to the function $s_{n}$. The idea of the proof is as follows: Our definition of inflection yields a natural concept of concavity for step functions. Within each interval of concavity we find suitable 
linear functions that bound $\left|s_{n}\right|$ from below as illustrated in Figure 9. Then we show that these bounds are comparable to the maximum $\left\|s_{n}\right\|_{\infty}$.

Basic assumption: Let the sign change of $S_{n}$ be located at $Z$, the extreme at $E$, and the inflection at $I$. We will assume that the extreme is a minimum and that $E<I$, the other cases being analogous by symmetry. Note that $S_{n-1}$ has a minimum at $I$ and a down-change at $E$. In particular, $S_{n-1}$ is negative to the right of $E$, so $S_{n-1}(0)=\frac{y}{-x} S_{n-1}(n-2)>0$. Then $S_{n-1}(1)<S_{n-1}(0)$ by property $(\mathrm{ShD})$, and $S_{n-1}(1)>0$ because it is to the left of the down-change.

Definition. To avoid carrying factors of $\frac{1}{n-1}$ we follow the convention that indices from 1 to $n-1$ are represented by capital letters, and their counterparts in the interval $[0,1]$ by the corresponding lowercase letter. In particular, we let $e:=\frac{E-1 / 2}{n-1}, i:=\frac{I-1 / 2}{n-1}$, and $z:=\frac{z-1 / 2}{n-1}$.

Definition. For given $n$, and integers $1 \leq A, B \leq n-1$, let $a:=\frac{A-1 / 2}{n-1}, b:=\frac{B-1 / 2}{n-1}$. We denote by $\Lambda_{n}\left(a, w_{1}, b, w_{2}\right)$ the linear function whose graph is the straight line from $\left(a, w_{1}\right)$ to $\left(b, w_{2}\right)$. Also, let $\mu(a, b)$ be the length $b-a$ of the interval $[a, b]$.

For our purposes, $w_{j}$ will always be 0 or $s_{n}(a)$ for some $a \in[0,1]$. Consequently, if $s_{n}$ is positive, and $s_{n-1}$ is increasing (so $s_{n}$ is "concave") from $a$ to $b$, the function $\Lambda_{n}\left(A, s_{n}(a), B, 0\right)$ is also positive in $(a, b)$. Moreover, its integral gives a lower bound for $\int\left|s_{n}\right|$ on every intermediate interval where $s_{n}$ is constant.
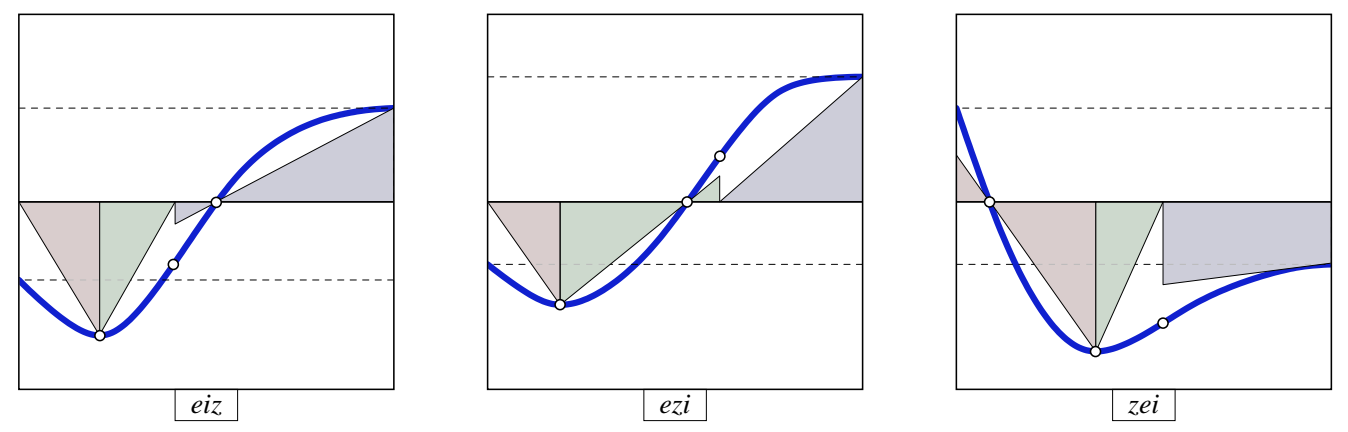

FiguRE 9. These continuous functions are caricatures of the step function $s_{n}$ in the different situations of cases 1, 2, 3. The 1-norm is bounded from below by the shaded areas under the linear functions. We show that these areas are comparable to the maximum $\left\|s_{n}\right\|_{\infty}$.

The function $s_{n}$ can adopt one of three forms depending on the order of $e, i$, and $z$. In each case we split $[0,1]$ into the same three intervals $[0, e],[e, i]$, and $[i, 1]$, and describe linear functions on these intervals that bound $\left|s_{n}\right|$ from below (compare Figure 9).

Case $1(e<i<z)$ : The linear functions are

(a) On $[0, e]: \Lambda\left(0,0, e, s_{n}(e)\right)$. The area of the triangle is

$$
\frac{1}{2} \cdot\left|s_{n}(e)\right| \cdot \mu(0, e)
$$

(b) On $[e, i]: \Lambda\left(e, s_{n}(e), i, 0\right)$. The area of the triangle is

$$
\frac{1}{2} \cdot\left|s_{n}(e)\right| \cdot \mu(e, i)
$$


(c) On $[i, 1]: \Lambda\left(z, 0,1, s_{n}(1)\right)$. The slope is $\frac{s_{n}(1)}{\mu(z, 1)}$ so the areas of the two triangles are

$$
\frac{\left|s_{n}(1)\right|}{\mu(z, 1)} \cdot\left(\frac{\mu(z, 1)^{2}}{2}+\frac{\mu(i, z)^{2}}{2}\right) \geq \frac{\left|s_{n}(1)\right|}{\mu(z, 1)} \cdot \frac{\mu(i, 1)^{2}}{4} \geq \frac{1}{4} \cdot\left|s_{n}(1)\right| \cdot \mu(i, 1)
$$

Case $2(e<z<i)$ : The linear functions are

(a) On $[0, e]: \Lambda\left(0,0, e, s_{n}(e)\right)$. The area of the triangle is

$$
\frac{1}{2} \cdot\left|s_{n}(e)\right| \cdot \mu(0, e)
$$

(b) On $[e, i]: \Lambda\left(e, s_{n}(e), z, 0\right)$. The slope is $\frac{\left|s_{n}(e)\right|}{\mu(e, z)}$. Since $a^{2}+b^{2} \geq(a+b)^{2} / 2$, the areas of the two triangles are

$$
\frac{\left|s_{n}(e)\right|}{\mu(e, z)} \cdot\left(\frac{\mu(e, z)^{2}}{2}+\frac{\mu(z, i)^{2}}{2}\right) \geq \frac{\left|s_{n}(e)\right|}{\mu(e, z)} \cdot \frac{\mu(e, i)^{2}}{4} \geq \frac{1}{4} \cdot\left|s_{n}(e)\right| \cdot \mu(e, i)
$$

(c) On $[i, 1]: \Lambda\left(i, 0,1, s_{n}(1)\right)$. The area of the triangle is

$$
\frac{1}{2} \cdot\left|s_{n}(1)\right| \cdot \mu(i, 1)
$$

$\underline{\text { Case } 3}(z<e<i)$ : The linear functions are

(a) On $[0, e]: \Lambda\left(z, 0, e, s_{n}(e)\right)$. The slope is $\frac{s_{n}(e)}{\mu(z, e)}$ so the areas of the two triangles are

$$
\frac{\left|s_{n}(e)\right|}{\mu(z, e)} \cdot\left(\frac{\mu(0, z)^{2}}{2}+\frac{\mu(z, e)^{2}}{2}\right) \geq \frac{\left|s_{n}(e)\right|}{\mu(z, e)} \cdot \frac{\mu(0, e)^{2}}{4} \geq \frac{1}{4} \cdot\left|s_{n}(e)\right| \cdot \mu(0, e)
$$

(b) On $[e, i]: \Lambda\left(e, s_{n}(e), i, 0\right)$. The area of the triangle is

$$
\frac{1}{2} \cdot\left|s_{n}(e)\right| \cdot \mu(e, i)
$$

(c) On $[i, 1]: \Lambda\left(a, s_{n}(a), b, s_{n}(b)\right)$, where $A=n-3, B=n-2$, and $a=\frac{A-1 / 2}{n-1}$, $b=\frac{B-1 / 2}{n-1}$. The region bounded by this $\Lambda$ consists of a rectangle of base $\mu(i, 1)$ and height $s_{n}(1)$, plus a triangle of base $\mu(i, 1)$ and slope $s_{n-1}(b)$. The area of $s_{n}$ is at least

$$
\left|s_{n}(1)\right| \cdot \mu(i, 1)+\left|s_{n-1}(b)\right| \cdot \frac{\mu(i, 1)^{2}}{2} .
$$

In each of the three cases, $\left\|s_{n}\right\|_{1}$ is bounded by a sum of three estimates. A trivial weakening of these expressions allows us to consolidate cases 1 and 2 into one:

1\&2: If $(e<z)$, then $\left\|s_{n}\right\|_{1} \leq \frac{1}{4}\left(\left|s_{n}(e)\right| \cdot \mu(0, i)+\left|s_{n}(1)\right| \cdot \mu(i, 1)\right)$.

3: If $(z<e)$, then $\left\|s_{n}\right\|_{1} \leq \frac{1}{4}\left(\left|s_{n}(e)\right| \cdot \mu(0, i)+\left|s_{n}(1)\right| \cdot \mu(i, 1)+\left|s_{n-1}(b)\right| \cdot \mu(i, 1)^{2}\right)$.

Note that $\left|s_{n}(e)\right| \geq\left|s_{n}(0)\right|=\frac{|x|}{y}\left|s_{n}(1)\right|$. If the interval $[0, i]$ has definite size, say $\mu(0, i)>$ $\frac{|x|}{6}$, then we can neglect the portion of the bounds that contains $\mu(i, 1)$ and see that

$$
\left\|s_{n}\right\|_{1} \geq \frac{1}{4} \frac{|x|}{6}\left|s_{n}(e)\right| \geq \frac{x^{2}}{24}\left|s_{n}(1)\right|
$$

in all three cases. Since $\left\|s_{n}\right\|_{\infty}$ is either $\left|s_{n}(e)\right|$ or $\left|s_{n}(1)\right|$, we find

$$
\left\|s_{n}\right\|_{1} \geq \frac{x^{2}}{24 y}\left\|s_{n}\right\|_{\infty} \text {. }
$$

To finish the proof we have to consider what happens when $\mu(0, i)<\frac{|x|}{6}$. In this situation we neglect the portion of the bounds that contains $\mu(0, i)$, and show that both $\left|s_{n}(1)\right|$ and 
$\left|s_{n-1}(b)\right|$ have lower bounds (24), (25), (26) of the form constant times $\left|s_{n}(e)\right|$. Just as above, this implies a lower bound for $\left\|s_{n}\right\|_{1}$ in terms of $\left\|s_{n}\right\|_{\infty}$, and we are done.

As a note of caution, note that for this final step we revert to the language of sequences $S_{n}$. Thus, instead of seeking bounds for $s_{n}(1), s_{n-1}(b)$ in terms of $s_{n}(e)$, we get equivalent bounds for $S_{n}(n-2), S_{n-1}(n-3)$ in terms of $S_{n}(E)$.

Lemma 17. If $I<\frac{|x| n}{6}$ then $\left|S_{n-1}(1)\right| \leq \frac{1}{2}\left|S_{n}(0)\right|$.

From Lemma 17 follows $\left|S_{n}(0)\right| \leq \frac{2}{3}\left|S_{n}(1)\right|$ (see (10)). Hence, if $\left|S_{n}(1)\right| \geq \frac{\left|S_{n}(E)\right|}{2}$, we get

$$
S_{n}(n-1)=\frac{y}{|x|}\left|S_{n}(0)\right| \geq \frac{2}{3} \frac{y}{|x|}\left|S_{n}(1)\right| \geq \frac{y}{3|x|}\left|S_{n}(E)\right| .
$$

It rests only to consider what happens when

$$
\left|S_{n}(1)\right| \leq \frac{\left|S_{n}(E)\right|}{2} .
$$

If this is the case, property (ShE) gives the following bound for cases 1 and 2:

$$
\frac{\left|S_{n}(1)\right|}{\mu((1-\eta), 1)} \geq \frac{y}{|x|} \frac{\left|S_{n}(E)-S_{n}(1)\right|}{\mu(0, E)} \geq \frac{y}{|x|}\left|S_{n}(E)-S_{n}(1)\right|
$$

where $\eta=\max \{I, Z\}$.

In case 3, property (ShE) gives a different bound:

$$
\left|S_{n-1}(n-3)\right| \geq \frac{y}{|x|} \frac{\left|S_{n}(E)\right|}{\mu(Z, I)} .
$$

Proof of Lemma 17. Throughout this section the basic assumption has been that $S_{n}(E)<0$ and $E<I$, so that $S_{n-1}$ has a minimum at $I$ and a down-change at $E$. Recall that this implies $S_{n-1}$ is negative to the right of $E$, and $0<S_{n-1}(1)<S_{n-1}(0)$.

First we derive Inequality (28). Since $y-x=1$ and $n / 2 \leq n-1$,

$$
I<\frac{-x n}{6}=\frac{-x(n / 2)}{2+y-x} \leq \frac{-x(n-1)}{2+y-x}
$$

So

$$
\frac{-x(n-1-I)}{(2+y) I} \geq 1 .
$$

The left expression increases if $I$ is replaced below by $E$ :

$$
\frac{-x(n-1-I)}{(2+y) E} \geq 1,
$$

giving

$$
-2 E \geq y E+x(n-1-I) .
$$

Now, the right expression is negative because it is smaller than $y I+x(n-1-I)=I+x(n-$ $1)<I+\frac{x n}{6}<0$, so dividing in (27) and multiplying by $y$ gives

$$
\frac{-y E}{y E+x(n-1-I)} \leq \frac{y}{2} \text {. }
$$


Using inequality (28) we prove Lemma 17 as follows. The absolute value of $S_{n-1}$ is decreasing from 1 to $E$, and from $I$ to $n-2$. In the second of these spans the average exceeds the rightmost term $S_{n-1}(n-2)$; thus,

$$
\begin{gathered}
\left|\sum_{j=1}^{E} S_{n-1}(j)\right|<E\left|S_{n-1}(1)\right|<E\left|S_{n-1}(0)\right|=E\left|\frac{y}{x} S_{n-1}(n-2)\right|< \\
\frac{y E}{|x| m}\left|\sum_{j=I}^{n-2} S_{n-1}(j)\right|<\frac{y E}{|x| m}\left|\sum_{j=E+1}^{n-2} S_{n-1}(j)\right| .
\end{gathered}
$$

where $m:=n-1-I$.

Substitute this estimate in

$$
\begin{aligned}
&\left|\sum_{j=1}^{n-2} S_{n-1}(j)\right|=\left|\sum_{j=E+1}^{n-2} S_{n-1}(j)\right|-\left|\sum_{j=1}^{E} S_{n-1}(j)\right| \geq \\
&\left(1-\frac{y E}{|x| m}\right)\left|\sum_{j=E+1}^{n-2} S_{n-1}(j)\right| .
\end{aligned}
$$

Now, using (29), (30), and (28),

$$
\begin{gathered}
\left|S_{n-1}(1)\right|<\left|\sum_{j=1}^{E} S_{n-1}(j)\right|<\frac{y E}{|x| m}\left|\sum_{j=E+1}^{n-2} S_{n-1}(j)\right|< \\
\frac{\frac{y E}{|x| m}}{\left(1-\frac{y E}{|x| m}\right)}\left|\sum_{j=1}^{n-2} S_{n-1}(j)\right|=\frac{-y E}{y E-|x| m}\left|\sum_{j=1}^{n-2} S_{n-1}(j)\right| \leq \frac{y}{2}\left|\sum_{j=1}^{n-2} S_{n-1}(j)\right|=\frac{1}{2}\left|S_{n}(0)\right| .
\end{gathered}
$$

\section{ACKNOWLEDGMENTS}

We wish to give thanks to F. Przytycki and the Polish Academy of Sciences for their hospitality at the conference center in Będlewo, Poland; to the Institut Mittag-Leffler for its hospitality during the final phase of this project; to M. Benedicks for conversations that led to the project this work stems from; and to P. Bleher for suggesting the use of integral operator theory as a tool for estimating asymptotic growth.

\section{REFERENCES}

[1] John B. Conway. A course in functional analysis, volume 96 of Graduate Texts in Mathematics. SpringerVerlag, New York, second edition, 1990.

[2] Frank Harary, John P. Hayes, and Horng-Jyh Wu. A survey of the theory of hypercube graphs. Comput. Math. Appl., 15(4):277-289, 1988.

[3] Tosio Kato. Perturbation theory for linear operators. Classics in Mathematics. Springer-Verlag, Berlin, 1995. Reprint of the 1980 edition.

[4] Michał Misiurewicz and Zbigniew Nitecki. Combinatorial patterns for maps of the interval. Mem. Amer. Math. Soc., 94(456):vi+112, 1991.

[5] N. J. A. Sloane. The on-line encyclopedia of integer sequences, 2010. Published electronically at www.research. att. com/ $\sim \mathrm{njas} /$ sequences/.

[6] Richard P. Stanley. Enumerative combinatorics. Vol. 2, volume 62 of Cambridge Studies in Advanced Mathematics. Cambridge University Press, Cambridge, 1999. With a foreword by Gian-Carlo Rota and appendix 1 by Sergey Fomin. 
LD-224R IUPUI, 402 N. Blackford St., Indianapolis, IN 46202, USA

E-mail address: rperez@math.iupui.edu 